\title{
Large-scale databases of proper names
}

\author{
PATRICK CONLEY, CURT BURGESS, and DOTTY HAGE \\ University of California, Riverside, California
}

\begin{abstract}
Few tools for research in proper names have been available-specifically, there is no large-scale corpus of proper names. Two corpora of proper names were constructed, one based on U.S. phone book listings, the other derived from a database of Usenet text. Name frequencies from both corpora were compared with human subjects' reaction times (RTs) to the proper names in a naming task. Regression analysis showed that the Usenet frequencies contributed to predictions of human RT, whereas phone book frequencies did not. In addition, semantic neighborhood density measures derived from the HAL corpus were compared with the subjects' RTs and found to be a better predictor of RT than was frequency in either corpus. These new corpora are freely available on line for download. Potentials for these corpora range from using the names as stimuli in experiments to using the corpus data in software applications.
\end{abstract}

Proper names present an interesting puzzle to psychology. Although very important for adequate social performance, for instance, memory for proper names is inferior to memory for other types of information (Cohen \& Burke, 1993). McWeeny, Young, Hay, and Ellis (1987) found that last names that connote some occupation (e.g., "Joe Baker went to the store") are more difficult to recall than if the actual occupation had been used ("The baker went to the store"). Burgess and Conley (1998) uncovered other interesting aspects of memory for proper names. They demonstrated, in the hyperspace analogue to language (HAL) semantic model of memory, that proper names cluster tightly in high-dimensional memory space, that ambiguity in proper names draws a name out of this pure name space toward words with more semantic depth, and that proper name semantic neighborhoods can encode information such as gender. It seems clear that proper names involve the storage and retrieval of important $\operatorname{cog}$ nitive information; however, it has only lately been an area of broader investigation. Three of the 11 chapters in the recent volume Corpus Processing for Lexical Acquisition deal with the issues involved in the representation of proper names (Boguraev \& Pustejovsky, 1996). Cohen and Burke edited a special issue of Memory that contained papers on the experimental, neuropsychological, and theoretical aspects of proper names. These examples provide evidence that research in proper names in all areas of psychology could be a rich field of inquiry.

This research was supported by a NSF Presidential Faculty Fellow Award SBR-9453406 to C.B. We thank Maureen Keeney and Janet Eakins for their work in data collection. More information about research at the Computational Cognition Lab, a HAL demo, and reprint information can be found at http://HAL.ucr.edu. Correspondence concerning this article should be addressed to P. Conley, Psychology Department, 1419 Life Sciences Bldg., University of California, Riverside, CA 92521-0426 (e-mail: patrick@cassandra.ucr.edu).
Unfortunately, the tools necessary to perform research in proper names are somewhat lacking. Specifically, no large-scale corpus of proper names is readily available for use in psychological research. The well-known Brown Corpus of Kučera and Francis (1967), for instance, only contains about 1,000 different first names, accounting for approximately 7,000 instances of proper (first) name use in the corpus. This is inadequate to generate name frequency experiments, for example, because many of the names appear only once in the Brown Corpus. To remedy this deficiency, we constructed proper name corpora from two very large databases, one derived from a structured list of proper names of American residents and the other derived from conversation-like text on Usenet newsgroups. Both of these main corpora of names were then also divided by gender. This information is now available to researchers for use in all forms of scientific inquiry and, in itself, may provide useful insights-for instance, how gender is represented or manifested on the Internet.

\section{AN EXAMINATION OF THE TWO CORPORA}

The first, and largest, corpus of proper names was derived from the residential phone book listing of all 50 states in the United States. The information was exported state by state from CD-ROM to text file format. ${ }^{l}$ Once processed, sorted, and tallied, the state files were combined into an omnibus file representing the proper name list for the entire country, consisting of over 80 million occurrences of proper names. We discovered that the 10,000 most frequent names accounted for over $85 \%$ of the total names that occurred more than once in the phone book corpus.

The second corpus of names was derived from the Usenet-based corpus used for training the HAL model of memory. The HAL Usenet corpus consists of approximately 320 million words drawn from the Usenet from 
the period of 1995-1997. All Usenet groups that contained text (i.e., binary groups were excluded) were included in this word corpus. The phone book corpus of proper names was then used to extract the corresponding proper name frequencies from the HAL Usenet corpus. Again, the most frequent names accounted for a large portion of the total number of names. In the Usenet proper names corpus, the 5,000 most frequent names accounted for over 70 million instances of proper names used on the Usenet.

The proper names corpus derived from the phone book frequencies has the advantage of being larger and more complete, as a substantial proportion of the nation's residents have a telephone and are listed in the local directory. The Usenet corpus of proper names has the advantage of being based on more conversational text and, therefore, ostensibly more closely represents the manner in which people actually employ proper names in daily use. It is interesting to note that female names are underrepresented in both corpora. As an example, in each corpus, the top 5,000 names accounted for approximately 70 million instances, but male names accounted for about $75 \%$ of the 70 million, whereas only $25 \%$ of the names were female. Possible explanations for a portion of this gender disparity range from greater use of abbreviations or more unlisted numbers for females in the phone book corpus to the male-dominated nature of the Internet.

\section{EXPERIMENT 1 \\ Comparisons of the Corpora With Human Reaction Times}

To test the hypothesis that, because of its conversational nature, the Usenet corpus of proper names is a better representation of the way people actually use proper names, the authors compared the frequency of names in both lists with human subjects' reaction time (RT) in a naming task to these names. It is well known that word frequency is one of the most reliable variables in predicting human RTs to words (see Chiarello, 1988, for a review) for both the lexical decision and the naming tasks. The initial access of memory information is strongly affected by frequency of occurrence: Higher frequency words are accessed more rapidly than lower frequency words (Becker, 1976; Morton, 1969). Although word frequency has also been implicated at later stages of retrieval (Balota \& Chumbley, 1984, 1985), it is likely that frequency as a basic mechanism plays its role at this stage of initial access. Thus, the importance of having some measure of frequency of proper names is critical in developing stimuli for experiments. In this experiment, we explore the degree to which Usenet and phone book corpora frequencies predict subjects' naming times to the proper names.

\section{Method}

Subjects. Naming times, or naming RTs, to experimental stimuli were gathered from undergraduate students enrolled in an introductory psychology class $(n=29)$, as part of a requirement for that course. All the subjects were native English speakers, with normal or corrected vision.

Stimuli. A quasi-randomly selected set of 238 first names and corresponding frequencies were extracted from the phone book proper names corpus. These same names and associated frequencies were then extracted from the Usenet proper names corpus. Despite the greater size and number of different names in the phone book corpus, only 4 names in the test set of 238 names did not appear in the Usenet corpus. The test set was made up of names from three different frequency groupings. The high-frequency names were taken from the 1 st to the 2,000 th most frequent names. The mediumfrequency names were taken from the 4,000 th to the 6,000 th most frequent names, and the low-frequency names were taken from the 8,000 th to the 10,000 th most frequent names. All frequency rankings for the purpose of word selection were based on frequencies from the phone book corpus. The three subgroups were also divided into high-, medium-, and low-frequency groups within each subgroup, so that nine different frequency ranges were represented. In addition, each range was balanced for gender so that equal numbers of male and female names were included in the test set. This classification by gender was conducted on the top 10,000 names in the corpora, with names sorted into four categories: male names, female names, gender-ambiguous names, and nonnames (e.g. titles such as Dr.). Only male and female names that were not gender ambiguous were used in this experiment. In each frequency range and gender condition, names were randomly selected from a list of possible names until the appropriate number of names for each condition was selected.

Procedure. The stimuli were presented to the subjects on a computer monitor. The subjects placed their chins on a chinrest, to standardize viewing distance and position. The subjects' responses to the stimuli were collected by microphone, which was connected to the presenting computer by a Digitry CTS system. Each trial in the experiment began with a plus sign appearing on a computer monitor as a central fixation point. This point was presented for $500 \mathrm{msec}$ and then was replaced by a proper name from the stimulus set in the same location. The proper name remained on the screen until the onset of the subjects' vocal response to the naming task, or until $2,000 \mathrm{msec}$ had passed with no vocal response. If no vocal response was recorded, a beep sounded and a time out message appeared on the screen. The subject was instructed to read the name aloud as quickly but as accurately as possible. The intertrial interval was $1 \mathrm{sec}$. The experimenter remained with the subject during the list presentation.

\section{Results and Discussion}

There was a strong relationship between the frequencies from the Usenet corpus and those from the phone book corpus ( $r=.88, p<.0001)$. This strong correlation is important, given the completely different nature of the two corpora involved, one drawn from a structured and formal listing of proper names and the other derived from Usenet postings. The overall mean RT was $721 \mathrm{msec}$. Mean RT was $640 \mathrm{msec}$ for the high-frequency group of names, $763 \mathrm{msec}$ for the medium-frequency group, and $757 \mathrm{msec}$ for the low-frequency group. Nonresponses accounted for only .009 of the trials, and these nonresponses were excluded from the analysis.

Despite the high correlation between the two corpora, the patterns of correlations between the proper names corpora and human RTs were very different. The correlations for the high-frequency groups were quite similar for the Usenet corpus $(r=-.21, p<.0001)$ and the phone 
book corpus $(r=-.19, p<.0001)$. Differences in the reliability of these relationships varied for the mediumand low-frequency proper names. Medium-frequency names from the Usenet corpus correlated reliably with RT $(r=-.21, p<.0001)$. However, the direction of relationship between name frequency and RT for the phone book corpus reversed direction $(r=.09, p<.001)$. Lowfrequency proper names from the Usenet corpus did not predict RT ( $r=-.03)$. The reversal of the expected correlation found with medium-frequency proper names from the phone book corpus was also seen with the lowfrequency proper names from the phone book $(r=.10$, $p<.0001)$. The omnibus correlations for each corpus were $r=-.19$ for the Usenet corpus and $r=-.16$ for the phone book corpus.

A stepwise multiple regression analysis was performed on the name frequency and RT data. The results in Table 1 demonstrate that, although the Usenet (HAL corpus) frequencies contributed significantly to the prediction of name RT, the phone book frequencies did not contribute anything further to the prediction of $\mathrm{RT}$-probably owing to'its correlation with the Usenet frequencies - and, thus, were not included in the model.

\section{EXPERIMENT 2 \\ Comparisons of the HAL Word Neighborhood Densities With Human Reaction Times}

Experiment 1 demonstrated that the relationship between name frequency in the corpora and RT was problematic, with the relationship growing increasingly obscure as the names dropped in frequency. Despite the fact that the correlations reversed direction or lost reliability at lower levels of frequency, the omnibus correlation for Usenet and phone book frequencies demonstrated the expected inverse relationship between the frequency of a name and RT to that name. In addition, the Usenet frequencies were demonstrated to be a better predictor of human RT than were phone book frequencies, especially at lower name frequencies.

Recent research, however, has demonstrated that density measures of a word's semantic space can be an even better predictor of word recognition than is frequency (Buchanan, Burgess, \& Lund, 1996; Burgess \& Conley, 1998). The HAL model of memory can successfully predict many different semantic and categorical effects (Burgess \& Lund, 1997; Lund \& Burgess, 1996). The HAL model of memory stores the semantic representations of words by using a lexical co-occurrence algorithm on a

Table 1

Summary of Stepwise Regression Analysis for Variables Predicting Reaction Time in a Naming Task

\begin{tabular}{|c|c|c|c|}
\hline Variable & $B$ & $S E B$ & $\beta$ \\
\hline \multicolumn{4}{|l|}{ Step 1} \\
\hline HAL (Usenet) frequency & -0.000003 & $0.00000 \mathrm{I}$ & -.19 \\
\hline
\end{tabular}

Note- $R^{2}=.036$ for Step $1(p<.0001)$. 320-million word corpus of Usenet text, which is very similar in nature to conversational speech. This process creates word meaning vectors that encode the semantic and contextual effects of words in a 140,000-dimensional meaning space on the basis of the context in which the words appear in a corpus of text. HAL creates these semantic representations by moving a 10 -word window over a corpus of text. Within this 10-word window, weights are assigned to words on the basis of how close they are to each other in the sentence. HAL stores this co-occurrence data in a $70,000 \times 70,000$ matrix, with a row and column for each of the 70,000 most frequent words in the corpusthe rows and columns representing the preceding cooccurrences and following co-occurrences, respectively. The row information is concatenated to the column information, producing a 140,000 -element vector for each word. This vector contains location information for the word in high-dimensional semantic space - in effect, the word's coordinates in the high-dimensional space generated by the matrix. The contextual or semantic similarity between 2 words is inversely proportional to their distance (computed with a Minkowski metric) in this space, meaning that semantically related words are closer together; words of similar grammatical class tend to cluster in this space as well (Burgess \& Lund, 1997). In a previous study, Burgess and Conley (1998) demonstrated that proper names cluster in their own area of high-dimensional space as well, accounting for some of the peculiarities in memory for proper names while contrasting with other models of proper name memory that postulate a special module or system for the storage and processing of proper names (i.e., Burton \& Bruce, 1993; Valentine, Bredart, Lawson, \& Ward, 1991). In addition, proper names had significantly greater densities than common nouns with the same frequency, meaning that proper names were closer to their neighbors in semantic space than frequencymatched common words were to their word neighbors. This suggests that word density in semantic space may play an important role in memory for proper names.

We were interested in determining how well semantic density measures from the HAL model of memory would predict human RT. It is known that word frequency plays a part in the location of words in high-dimensional space, but the HAL model has repeatedly demonstrated that much more than mere frequency is involved in the topography of high-dimensional space (Audet \& Burgess, 1998; Burgess \& Conley, 1998). As HAL word densities have been demonstrated to be an index of both semantic depth and word difficulty, as well as modeling negative effects such as those found in aging (Conley \& Burgess, in press), these word densities might, therefore, be better predictors of human RTs than are word frequencies.

\section{Method}

In this experiment, we reanalyzed the RT data gathered in Experiment 1 by comparing RTs with HAL word densities. The highdimensional densities were operationalized as the distance to the nearest neighbor to each name in high-dimensional space. Because 
Table 2

Summary of Stepwise Regression Analysis for Variables Predicting Reaction Time in a Naming Task

\begin{tabular}{lccr}
\hline \multicolumn{1}{c}{ Variable } & $B$ & $S E B$ & $\beta$ \\
\hline $\begin{array}{l}\text { Step 1 } \\
\text { HAL densities }\end{array}$ & 0.000348 & 0.000028 & .27 \\
Step 2 & & & \\
$\quad$ HAL densities & 0.000302 & 0.000031 & .23 \\
Phone book frequency & -0.000001 & 0.000001 & -.07 \\
\hline Note- $R^{2}=.072$ for Step 1; $\Delta R^{2}=.005$ for Step 2 $(p \mathrm{~s}<.001)$.
\end{tabular}

only the 70,000 most frequent word vectors are stored in the HAL memory matrix, only 138 of the 238 names used in Experiment 2 were represented in the matrix. Thus, densities could only be gathered for this smaller set of names.

\section{Results and Discussion}

The correlation between high-dimensional density and RT was stronger than the corresponding correlation between word frequency and RT. Because of the truncation of the names list, owing to the HAL model's limit of 70,000 most frequent words, only an omnibus correlation was calculated $(r=.27, p<.0001)$. This correlation is higher than the previously reported omnibus correlations for the Usenet corpus $(r=-.19)$ and for the phone book corpus $(r=-.16)$, and also is larger than the largest correlations reported in the previous study, those for the highfrequency group of proper names $(r=-.21$ and $r=$ -.19 for Usenet and phone book corpora, respectively). The direction of the relationship is reversed for the current correlation, because RT is now being compared with distance in high-dimensional space. The correlation between HAL density and name frequency was $r=-.56$ for the Usenet corpus and $r=-.45$ for the phone book corpus.

Again, a stepwise regression analysis was performed, this time with the HAL density variable added to the previous variables of Usenet and phone book frequency in predicting RT. As Table 2 reveals, when HAL density was added to the regression equation, it was by far the best predictor, with only a slight improvement in prediction when the phone book frequency variable was accounted for. HAL frequency, the only significant variable in Experiment 1 , was no longer reliable when HAL densities were accounted for, likely owing to its correlation to the density metric.

\section{GENERAL DISCUSSION}

Our results show that the HAL density metric was the best possible predictor of RT. However, when the HAL density metric was not accounted for or not available, Usenet frequency was a better predictor of RT than was phone book frequency. This finding probably reflects the more conversational nature of the Usenet text, whereas the phone book corpus is derived from a more structured listing, with more formal forms of names listed and fewer nicknames and diminutives employed.

Another important finding was that the frequency of a proper name was not nearly as good a predictor of RT as has been found to occur with other classes of words, such as common nouns. For example, in Burgess and Livesay (1998), the correlations between common noun frequency (derived from the HAL corpus) and RT were twice as large as the correlations reported in this study. The lesser role of word frequency in predicting RT is yet another example of how memory for proper names has different characteristics than memory for other types of information (see Burgess \& Conley, 1998).

The correlations involved in these experiments are not large, and the various measures (Usenet frequency, phone book frequency, and HAL word density) correlate more highly with each other than they do with human RT to proper names. Considering the noisy and problematic nature of the corpora, this result may not be surprising. For instance, many proper names are ambiguous with other word classes, and this name/word ambiguity very likely has an effect on RT (see Table 3 for examples). We believe, however, that this name/noun ambiguity is actually an advantage for the Usenet corpus. Each exposure to an ambiguous name/noun (such as bill) should increase a person's familiarity with that word, resulting in a corresponding decrease in RT. Thus, Usenet better models the wide variety of uses and ambiguous situations in which names are employed in natural language. Actual usage information has been shown to be important in characterizing the memory retrieval process with ambiguous words, particularly the initial lexical access (Simpson \& Burgess, 1985).

Also, although the overall correlations between phone book and Usenet corpora are similar, Usenet was a better predictor for the low and medium name frequency conditions. In fact, the phone book frequencies reversed direction. The superiority of the Usenet corpus in predicting lower frequency words has been noted in previous studies (Burgess \& Livesay, 1998). As more research focuses on RT and other measures of cognitive performance with low-frequency words as experimental stimuli, the better predictive abilities of the Usenet corpus lead the authors to recommend the Usenet corpus in cases in which low-frequency names are being considered.

Despite the experiments in this study, our main purpose in comparing the proper names corpora with RTs was to emphasize the different origin and nature of the two corpora. Proper names research is a relatively new field, but a field that has seen rapid growth. The experi-

Table 3

Examples of Ambiguous High-Frequency Names

$\begin{array}{ll}\text { jack } & \text { rose } \\ \text { rob } & \text { sue } \\ \text { mark } & \text { dawn } \\ \text { frank } & \text { peg } \\ \text { bill } & \text { ruby } \\ \text { art } & \text { grace } \\ \text { don } & \text { heather } \\ \text { bob } & \text { hazel } \\ \text { will } & \text { joy } \\ \text { curt } & \text { pearl } \\ \text { gene } & \text { holly }\end{array}$


mental stimuli normally used in this type of experiment, such as Kučera and Francis (1967), are not good sources of proper name information, especially first names such as those used in the present study. Either name corpus, as well as the associated corpus of HAL densities, is a potentially valuable source for information, both for future proper names research and in more practical applications, such as information retrieval applications. Which corpus should be used depends, of course, on the research or application. ${ }^{2}$

\section{REFERENCES}

AudeT, C., \& Burgess, C. (1998). Distinguishing between manner of motion and inherently directed motion verbs using a high-dimensional memory space and semantic judgments. In M. A. Gernsbacher \& S. J. Derry (Eds.), Proceedings of the Cognitive Science Society (pp. 6671). Hillsdale, NJ: Erlbaum.

Balota, D. A., \& Chumbley, J. I. (1984). Are lexical decisions a good measure of lexical access? The role of word frequency in the neglected decision stage. Journal of Experimental Psychology: Human Perception \& Performance, 10, 340-357.

Balota, D. A., \& Chumbley, J. I. (1985). The locus of word-frequency effects in the pronunciation task: Lexical access and/or production? Journal of Memory \& Language, 24, 89-106.

BECKER, C. A. (1976). Allocation of attention during visual word recognition. Journal of Experimental Psychology: Human Perception \& Performance, 2, 556-566.

Boguraev, B., \& Pustejovsky, J. (Eds). (1996). Corpus processing for lexical acquisition. Cambridge, MA: MIT Press.

Buchanan, L., Burgess, C., \& Lund, K. (1996). Overcrowding in semantic neighborhoods: Modeling deep dyslexia. Brain \& Cognition, 30, 111-114.

Burgess, C., \& CONLEY, P. (1998). Developing semantic representations for proper names. In M. A. Gernsbacher \& S. J. Derry (Eds.), Proceedings of the Cognitive Science Society (pp. 185-190). Hillsdale, NJ: Erlbaum.

Burgess, C., \& Livesay, K. (1998). The effect of corpus size in predicting reaction time in a basic word recognition task: Moving on from Kučera and Francis. Behavior Research Methods, Instruments, \& Computers, 30, 272-277.
Burgess, C., \& LUND, K. (1997). Modelling parsing constraints with high-dimensional context space. Language \& Cognitive Processes, 12, $177-210$.

Burton, A. M., \& Bruce, V. (1993). Naming faces and naming names: Exploring an interactive activation model of person recognition. Memory, 1, 457-480

Chiarello, C. (1988). Lateralization of lexical processes in the brain: A review of visual half-field research. In H. A. Whitaker (Ed.), Contemporary reviews in neuropsychology (pp. 36-76). New York: Springer-Verlag.

Cohen, G., \& Burke, D. M. (1993). Memory for proper names: A review. Memory, 1, 249-263.

Conley, P., \& Burgess, C. (in press). Age effects in a computational model of memory. Brain \& Cognition.

KuČERA, H., \& FRANCIS, W. N. (1967). Computational analysis of presentday American English. Providence, RI: Brown University Press.

Lund, K., \& Burgess, C. (1996). Producing high-dimensional semantic spaces from lexical co-occurrence. Behavior Research Methods, Instruments, \& Computers, 28, 203-208.

McWeeny, K. H., Young, A. W., Hay, D. C., \& Ellis, A. W. (1987). Putting names to faces. British Journal of Psychology, 78, 143-149.

MORTON, J. (1969). Interaction of information in word recognition. Psychological Review, 76, 165-178.

Simpson, G. B., \& BURGEss, C. (1985). Activation and selection processes in the recognition of ambiguous words. Journal of Experimental Psychology: Human Perception \& Performance, 11, 28-39.

Valentine, T., Bredart, S., LaWson, R., \& Ward, G. (1991). What's in a name? Access to information from people's names. European Journal of Cognitive Psychology, 3, 147-176.

\section{NOTES}

1. The phone book CD-ROM used in compiling the name frequency information was the 1996 Home Phone Book from ProCD. ProCD is located at 222 Rosewood Drive, Davers, MA 01923-4520, and their web page is http://www.procd.com/

2. Summary data for the two corpora, in addition to HAL model word densities, will soon be available from the authors' lab web page (http://hal.ucr.edu). The corpora themselves are too large to download through the Internet but will be available in the future on CD-ROM.

(Manuscript received December 15, 1998; revision accepted for publication March 24, 1999.) 